\title{
Harvesting Age and Time Effect of Andrographis paniculata Leaf on Its Anti-Inflammatory Activity
}

\author{
Azizul Isha1, Nur A'thifah Yusof', Intan Safinar Ismail1,2* \\ ${ }^{1}$ Laboratory of Natural Products, Institute of Bioscience, Universiti Putra Malaysia, Serdang, Malaysia \\ ${ }^{2}$ Department of Chemistry, Faculty of Science, Universiti Putra Malaysia, Serdang, Selangor Darul Ehsan, Malaysia \\ Email: *safinar@upm.edu.my
}

How to cite this paper: Isha, A., Yusof, N.A. and Ismail, I.S. (2016) Harvesting Age and Time Effect of Andrographis paniculata Leaf on Its Anti-Inflammatory Activity. Journal of Biosciences and Medicines, 4, 175-188.

http://dx.doi.org/10.4236/jbm.2016.412021

Received: May 16, 2016

Accepted: December 23, 2016

Published: December 26, 2016

Copyright $\odot 2016$ by authors and Scientific Research Publishing Inc. This work is licensed under the Creative Commons Attribution International License (CC BY 4.0).

http://creativecommons.org/licenses/by/4.0/

\begin{abstract}
In this study, Fourier Transform Infrared (FTIR)-, ${ }^{1} \mathrm{H}$ Nuclear Magnetic Resonance (NMR)-spectroscopy and multivariate statistical analysis were used for the screening of in vitro anti-inflammatory activity on three different germplasm accessions namely 11,341 (P), 11,248 (T) and 11,265 (H) of Malaysian Andrographis paniculata (A. paniculata) leaf. The anti-inflammatory activity by nitric oxide (NO) inhibition assay in relation to the different harvesting ages and times of $A$. paniculata leaf was determined through comparison by partial least-squares analysis (PLS) using SIMCA-P. The PLS allowed the separation and correlation between the NO assay with the phytochemical present due to the effects of different harvesting ages and times. From the PLS plots, conclusions were drawn with respect to the correlation between $A$. paniculata leaf metabolites with the anti-inflammatory results which showed that 180 days after transplanting (DAT) of morning session for accessions $\mathrm{T}$ and $\mathrm{H}$, and evening for $P$ gave the highest anti-inflammatory activity.
\end{abstract}

\section{Keywords}

Andrographis paniculata, Anti-Inflammatory, NO Inhibition, Partial Least-Squares Analysis, FTIR, ${ }^{1} \mathrm{H}$ NMR

\section{Introduction}

Andrographis paniculata (Burm. F.) Nees or "Hempedu Bumi" belongs to the family Acanthaceae which is widely grown in tropical areas of Asia such as Malaysia, India, Pakistan and Sri Lanka [1]. A . paniculata is a traditional Chinese, Southeast Asian and Indian herb and has been of interest in research due to its wide uses in traditional medicine, for example: as anticancer, anti-inflammatory, anti-allergic, immuno-stimulatory, antithrombotic, hypoglycemic and hepatoprotective agent [2]-[8]. Because of its 
vast and interesting biological activities, it is desirable to phytochemically investigate this plant in details in order to better understand the responsible bioactive metabolites.

Recently, several reports on the bioactivities of $A$. paniculata particularly anti-inflammation have been published [9] [10]. However, the influence of $A$. paniculata harvesting ages and times on the anti-inflammatory activity has not been discussed. The optimization of the harvest age and time is crucial to standardize the quality of $A$. paniculata based on the major compounds content, i.e. andrographolide as it was reported to be decreasing with the delay in harvesting [11]. Therefore, the anti-inflammatory activity related to $\mathrm{NO}$ inhibition of $A$. paniculata leaf at different harvesting ages and times in correlation with its overall biomarkers was determined using metabolomics approach as described herein.

\section{Materials and Methods}

\subsection{Solvents and Chemicals}

Deuterated methanol- $d_{4}\left(\mathrm{CH}_{3} \mathrm{OH}-d_{4}\right)$, non-deuterated $\mathrm{KH}_{2} \mathrm{PO}_{4}$, sodium deuterated oxide $(\mathrm{NaOD})$, trimethylsilyl propionic acid- $d_{4}$ sodium salt (TSP), deuterium oxide $\left(\mathrm{D}_{2} \mathrm{O}\right)$ and methanol were purchased from Merck (Darmstadt, Germany). Liquid nitrogen was supplied by MOX-Linde Sdn. Bhd. (Petaling Jaya, Malaysia). Standard compounds such as andrographolide, neoandrographolide and deoxyandrographolide were previously isolated by Professor Dr. Johnson Stanslas from Pharmacotherapeutics Unit, Department of Medicine, Faculty of Medicine and Health Sciences, Universiti Putra Malaysia. RAW264.7 cells were purchased from American Type Culture Collection (ATCC, Rockville, USA) under the catalogue number of ATCC ${ }^{\oplus}$ TIB-71TM. Dulbecco's Modified Eagle's Medium (DMEM) with and without phenol red, fetal bovine serum (FBS), penicillin streptomycin (Pen Strep) and TrypLETM Express were purchased from Gibco by Life Technologies Inc. (Eggenstein, Germany). Phosphate buffer saline (PBS) was purchased from Invitrogen by Life Technologies. Lipopolysaccharides (LPS) from Escherichia coli 0111:B4 and interferon-gamma (IFN- $\gamma$ ) were purchased from Sigma Aldrich Co. (St. Louis, MO). DMSO was purchased from Merck (Darmstadt, Germany). PBS and 3-(4,5-dimethylthiazol-2-yl)-2,5-diphenyltetrazolium bromide (MTT) were purchased from Invitrogen by Life Technologies Inc. (Eggenstein, Germany) and DMSO was purchased from Merck (Darmstadt, Germany).

\subsection{Plant Materials and Cultivation}

The $A$. paniculata seeds from three germplasm accessions of Malaysia (Accession 11,265, 11,341 and 11,248 which will be designated as $\mathrm{H}, \mathrm{P}$ and $\mathrm{T}$, respectively) were collected in July 2011 from Ladang Dua, Universiti Putra Malaysia (UPM). The germination and cultivation procedure in this study was performed according to Yusof et al. (2014) [12]. Leaf samples were harvested in January, February and March 2012 assigned as 120, 150 and 180 DAT at two different time sessions (morning: 7:30 - 10:30 am; evening: 2:30 $5.30 \mathrm{pm}$ ). Each sample was quenched with liquid nitrogen and ground into powder form before stored at $-80^{\circ} \mathrm{C}$ prior to drying by freeze-dry system (Labconco, Texas, USA) to 
remove the water content.

\subsection{FTIR Sample Preparation and Measurement}

The FTIR sample preparation and measurement in this study were performed according to Yusof et al. (2014) [12]. The freeze-dried leaf powder of $A$. paniculata (150 mg) was extracted by sonication in $5 \mathrm{~mL}$ commercial-grade methanol $\left(30 \mathrm{~min}, 40^{\circ} \mathrm{C}\right.$ ). The extraction step was repeated twice and the total combined supernatant was filtered through filter paper (Whatman, $125 \mathrm{~mm}$ ) and evaporated using miVac Modular Concentrator series (Genevac Ltd, Ipswich, UK). The crude extracts were then kept at $-80^{\circ} \mathrm{C}$ until IR analysis. The crude samples were freeze-dried again before submitting to IR analysis to prevent absorbance interference caused by the water content. IR spectra were recorded using a Spectrum 100 FTIR spectrometer (PerkinElmer, Waltham, MA, USA) with a single reflectance horizontal ATR cell equipped with a zinc selenide crystal. The analyses were made in absorption mode on a small amount of crude sample deposited on the ATR crystal. The spectra of five biological replicates and pure compounds in three technical replicates were obtained in the frequency range $4000-280$ $\mathrm{cm}^{-1}$ with a resolution of $4 \mathrm{~cm}^{-1}$ and a total accumulation of 16 scans.

\subsection{NMR Sample Preparation and Measurement}

Freeze-dried plant leaf $(20 \mathrm{mg})$ was transferred to a $2.0 \mathrm{~mL}$ Eppendorf tube. A volume of $1.5 \mathrm{~mL}$ of a mixture of $\mathrm{KH}_{2} \mathrm{PO}_{4}$ buffer ( $\mathrm{pH} 6.0$ ) in $\mathrm{D}_{2} \mathrm{O}$ containing $0.05 \%$ trimethylsilylpropionic acid sodium salt (TSP, w/w) and methanol-d4 (1:1) was added to the plant sample. The extraction procedure in this study was performed according to Kim et al. (2010), with a minor modification [13]. The Eppendorf tubes were vortexed for 1 minute at room temperature and ultrasonicated for 20 minutes. This step was followed by centrifuging for 10 minutes at 10,000 rpm to remove the insoluble components from which a $0.75 \mathrm{~mL}$ clear supernatant was transferred into the $5 \mathrm{~mm}$ NMR tube. ${ }^{1} \mathrm{H}$ NMR spectra were recorded at $26^{\circ} \mathrm{C}$ on a $500 \mathrm{MHz}$ Varian INOVA NMR spectrometer operating at a proton NMR frequency of $499.887 \mathrm{MHz}$. The acquisition time of each ${ }^{1} \mathrm{H}-\mathrm{NMR}$ measurement was 3.53 minutes, which consisted of 64 scans. A presaturation sequence was used to suppress the residual $\mathrm{H}_{2} \mathrm{O}$ signal. The resulting spectra were manually phased and baseline was corrected using the Chenomx software (v.5.1, Alberta, Canada) with a consistent setting for all spectra. Spectral intensities were binned by equal width ( $\delta 0.04)$ corresponding to the region of $\delta 0.50-10.00$. The region of $\delta 4.70-4.90$ representing water, and $\delta 3.23-3.36$ representing residual methanol were excluded. 2D $J$-resolved was recorded for additional support in the metabolite identification.

\subsection{Relative Quantification of Targeted Profiling Data}

Andrographolide and neoandrographolide were selected from the ${ }^{1} \mathrm{H}$ NMR spectra and their concentrations are listed in Table 1. Deoxyandrographolide, $\alpha$-glucose, $\beta$-glucose, sucrose, choline and analine were excluded from the quantification due to their overlapping in the $1 \mathrm{D}$ NMR spectra. Relative quantification of the identified metabolites 
Table 1. Chemical shift assignment of ${ }^{1} \mathrm{H}$ NMR signals characteristics of selected metabolites in $A$. paniculata leaf extract and $p$ value of one-way ANOVA.

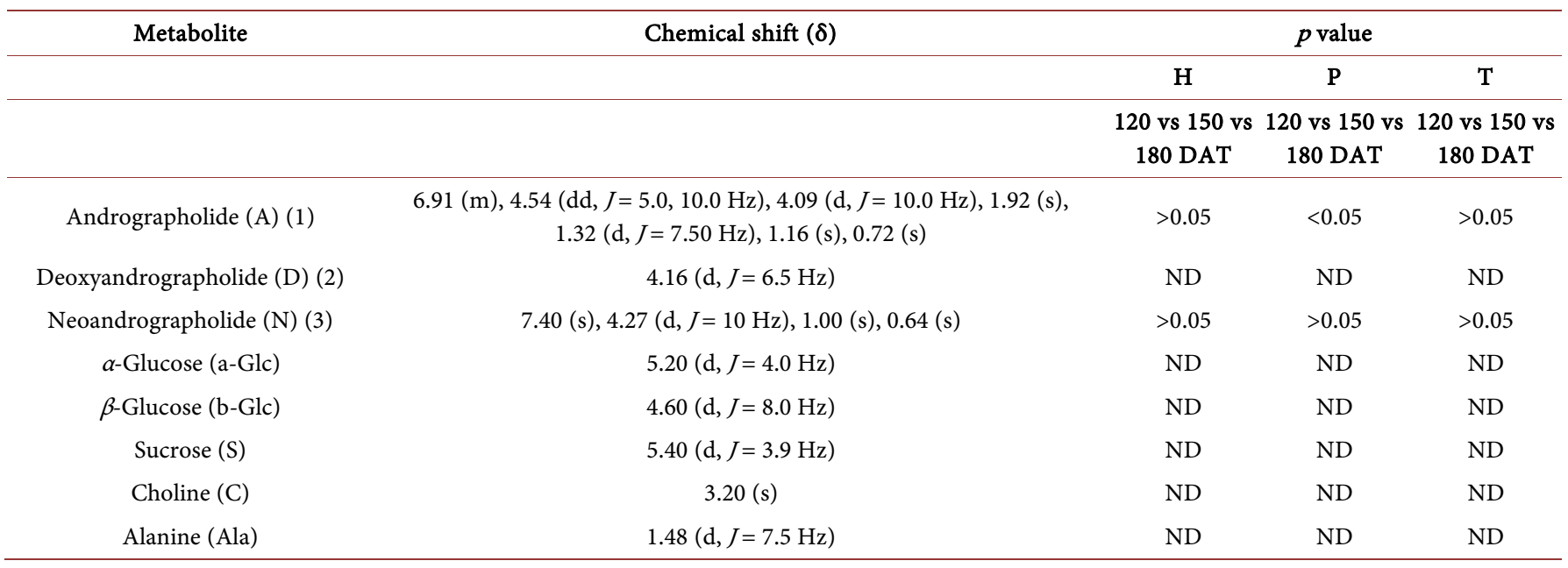

from the $A$. paniculata was based on the mean peak area of the ${ }^{1} \mathrm{H}$ NMR signal of interest after binning using Chenomx software. Data was analyzed using the Minitab Statistical Software 14.12.0, () 2004 Minitab Inc. PA 16801-9928, USA. Among the groups, significance test was performed using the one-way ANOVA at 5\% significance level.

\subsection{Nitric 0xide Assay}

The anti-inflammation activity of $A$. paniculata crude extracts was evaluated through Nitric oxide (NO) suppression activity based on Lee's method with some modifications [14]. Each $A$. paniculata crude extract of $2 \mathrm{mg}$ was dissolved in $5 \mu \mathrm{L}$ of DMSO/995 $\mu \mathrm{L}$ DMEM without phenol red. It was then sonicated for 10 minutes without heating, and centrifuged for 10 minutes at $1000 \mathrm{rpm}$. The solubility of $50 \%$ for the methanol crude extract was determined earlier as to estimate the actual concentration of the prepared crude extracts. From the resulted supernatant, $260 \mu \mathrm{L}$ was pipetted out into $1040 \mu \mathrm{L}$ DMEM without phenol red as to minimize the use of DMSO in the sample preparation. This is the stock extract which was further diluted into desired concentrations via serial dilutions. Two stock concentrations of 1000 and $10,000 \mu \mathrm{g} / \mathrm{mL}$ were subjected to the two-fold dilution in the screening stage. For the actual assay, the concentrations of $A$. paniculata extracts used were in the range of $100 \mu \mathrm{g} / \mathrm{mL}-1 \mathrm{mg} / \mathrm{L}$ in a serial dilution.

DMSO of $0.1 \%$ in DMEM without phenol red was utilized as a control vehicle in the serial dilutions in order to maintain the final concentration of $0.1 \%$ DMSO in each well. These various concentrations were prepared separately in the aseptic microcentrifuge tubes before being dispensed into the wells.

\subsection{Cell Culture and Treatment}

The RAW264.7 murine macrophages were cultured in $75 \mathrm{~cm}^{2}$ plastic culture flask in DMEM with phenol red containing HEPES, L-glutamine supplemented with 10\% FBS and $1 \%$ penicillin/streptomycin in $5 \% \mathrm{CO}_{2}$ atmosphere at $37^{\circ} \mathrm{C}$. After the cells have 
reached the confluency of $80 \%-90 \%$, they were detached from the flask wall by rinsing off the flask with $5 \mathrm{ml}$ of PBS solution followed by $2 \mathrm{ml}$ of TrypLE ${ }^{\mathrm{TM}}$ Express before incubated for 10 minutes. DMEM with phenol red of $4 \mathrm{~mL}$ was used to rinse out the detached cells which then centrifuged at $3000 \mathrm{rpm}, 4^{\circ} \mathrm{C}$ for 10 minutes by using Rotina 35R (Andreas Hettich GmbH \& Co., Tuttlingen, Germany). The supernatant was then removed and the cells were re-suspended with fresh DMEM containing HEPES, and L-glutamine with 5\% FBS but without phenol red. The cells were counted by using a standard trypan blue cell counting technique.

The cell concentration was adjusted to $1 \times 10^{5} \mathrm{cells} / \mathrm{mL}$ in the same medium (DMEM without phenol red) before seeded in a 96-well flat bottom tissue culture plates at initial seeding densities of $5 \times 10^{4}$ cells $/ 50 \mu \mathrm{L}$ per well. The ready plates with seeded cells were incubated at $37^{\circ} \mathrm{C}\left(5 \% \mathrm{CO}_{2}\right.$ atmosphere) overnight to allow anchoring of cells to the wells.

The treatment of the cells was done by replenishing the medium in each well with 50 $\mu \mathrm{L}$ DMEM without phenol red containing triggering agent of $1 \mathrm{ng} / \mathrm{mL}$ recombinant murine IFN $-\gamma$ and $1 \mu \mathrm{g} / \mathrm{mL}$ LPS. For each dilution of the plant extracts, $50 \mu \mathrm{L}$ was added into the appropriate wells in four replicates. The assay plate was incubated for 17 hours at $37^{\circ} \mathrm{C}$ in $5 \% \mathrm{CO}_{2}$ atmosphere. There were five kinds of controls prepared in duplicates for each plate. The controls were media only without cells (blank control), cells in media only, cells in media containing triggering agent plus $0.1 \%$ DMSO (negative control), cells in media containing triggering agent only, and cells containing triggering agents plus curcumin (positive control).

\subsection{Nitrite Determination}

Nitrite concentration was determined by Griess assay through the reaction of $50 \mu \mathrm{L}$ of cell culture supernatant and $50 \mu \mathrm{L}$ of Griess reagent (1\% sulphanilamide and $0.1 \%$ $N$-(1-naphthyl)ethylenediamine dihydrochloride in $2.5 \%$ phosphoric acid) at room temperature. The optical density was measured at $550 \mathrm{~nm}$ after 5 minutes of incubation at room temperature by Spectramax Plus (Molecular Devices) UV/Vis microplate reader. The accumulated NO release was determined spectrophotometrically by the total nitrite in the medium. The nitrite concentration in the samples was determined by regression analysis using serial dilutions of sodium nitrite as a standard. The percentage inhibition was calculated based on the ability to inhibit nitrite below the level produced by cells cultured in the presence of triggering agents and DMSO, which was considered as $0 \%$ inhibition.

\subsection{MTT Cell Viability Assay}

In order to avoid any false positive result of $\mathrm{NO}$ inhibition, the treated cells were also submitted for MTT assay as to measure any cytotoxic effect on the cells by using the Heras's method [15]. Supernatant in all wells was removed followed by the addition of $100 \mu \mathrm{L}$ DMEM with phenol red. Subsequently, $20 \mu \mathrm{L}$ of 3-(4,5-dimethylthiazol-2-yl)2,5-diphenyltetrazolium bromide (MTT, $5 \mathrm{mg} / \mathrm{ml}$ ) was added to each well and the plate 
was incubated in $5 \% \mathrm{CO}_{2}$ atmosphere at $37^{\circ} \mathrm{C}$ for $4 \mathrm{~h}$. The mixture of culture media and MTT in all wells were discarded and the purplish formazan crystals formed were dissolved in DMSO and further incubated for 5 minutes. The absorbance of colour intensity was then measured at $570 \mathrm{~nm}$ at the room temperature wherein the absorbance of formazan in the control of untreated cells was taken as $100 \%$ viability.

\subsection{Statistical Analysis}

Data were analyzed by using GraphPad Prism software version 5.01 (GraphPad Software Inc., San Diego, USA). The graph of half maximum inhibitory concentration ( $\mathrm{IC}_{50}$ ) for each sample was obtained from logarithm graph plot. In which each graph showed the goodness of fit, $R^{2}>0.95$ and within the $95 \%$ of the confidence interval with $p$-values $<0.05$ were considered significant. All results were expressed as the mean \pm S.E.M of $n$, where $\mathrm{n}$ represented the number of wells replication. Differences between the mean values for different groups were analyzed by Analysis of variance (ANOVA) using SPSS 16.0 (Statistical Package for the Social Sciences).

\subsection{Multivariate Data Analysis}

Multivariate data analysis by partial least-squares analysis (PLS) was performed with the SIMCA 13.0 software using scaling based on Variance.

\section{Results and Discussion}

\section{1. ${ }^{1} \mathrm{H}$ NMR Spectra and Metabolite Identification}

In the present work, metabolomics analysis was applied to discriminate the metabolite variation among the three accessions of $\mathrm{P}, \mathrm{T}$ and $\mathrm{H}$. Metabolites from this plant including the major andrographolide, deoxyandrographolide, neoandrographolide and carbohydrates ( $\alpha$-glucose, $\beta$-glucose and sucrose) as well as amino acids (alanine and choline) were successfully identified. Figure 1 shows the ${ }^{1} \mathrm{H}-\mathrm{NMR}$ spectra of $A$. paniculata leaf extracts displaying both the qualitative and quantitative variations of some metabolites. The compounds were identified based on the 2D J-resolved (Figure 2) and comparison with the reported NMR signals of those isolated metabolites from this plant. Other compounds were assigned by comparison with the NMR spectra of the reference compounds measured under the same conditions as extracts. All of the spectra showed signals in the aliphatic $(\delta 0.5-3.0)$, aromatic $(\delta 5.5-8.0)$ and carbohydrate region $(\delta$ $3.0-5.5)$.

Table 1 lists the characterized metabolites which could be recognized by signals shown in those three main regions. The peaks in at $\delta 0.64$ and $\delta 1.00$ were typical signals of the neoandrographolide aliphatic protons, whereas andrographolide signals were detectable at $\delta 0.72,1.16,1.32$ and 1.92. Some amino acids namely alanine and choline were also observed in the aliphatic region at 1.48 and 3.20, respectively. Alpha-glucose, $\beta$-glucose and sucrose were ascribed based on the signals displayed in the carbohydrate region of $\delta 3.0$ - 5.5. Other andrographolide signals were observed at $\delta 4.09$ and 4.54. As for deoxyandrographolide, the signal was detectable at $\delta 4.16$. Andrographolide and 


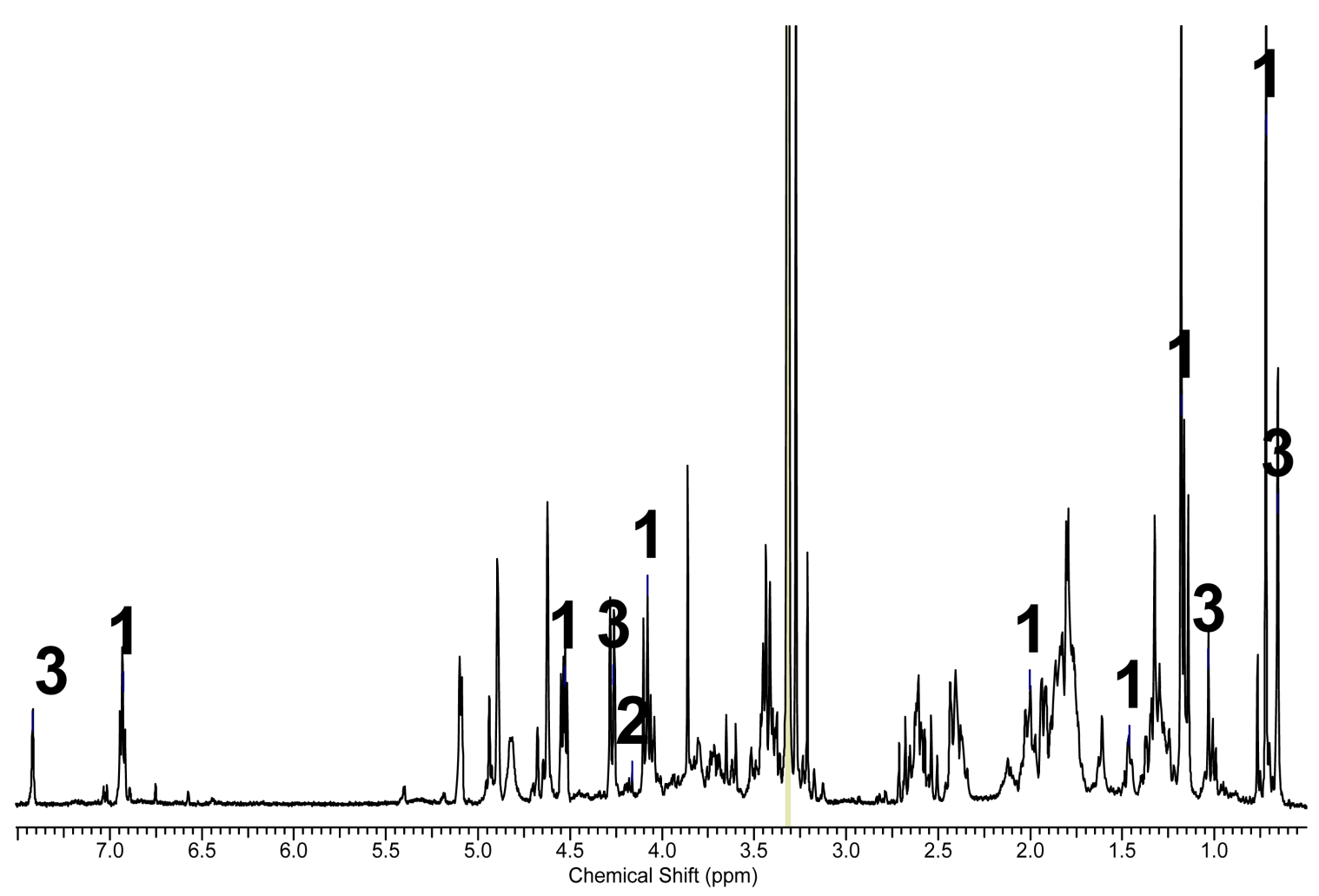

Figure $1 .{ }^{1} \mathrm{H}$ NMR spectra of the $A$. paniculata leaves extract: expanded range $\delta 0.50-7.50$. The observed signal as follows: 1 -andrographolide; 2-deoxyandrographolide and 3-neoandrographolide.

neoandrographolide signals were also observed in the aromatic region of $\delta 6.91$ and 7.40 respectively.

\subsection{Relative Quantification}

Relative quantification of the identified major compounds which are andrographolide and neoandrographolide in $A$. paniculata extracts was based on the mean peak area of the ${ }^{1} \mathrm{H}$ NMR signals from the three different accessions ( $\mathrm{P}, \mathrm{T}$ and $\left.\mathrm{H}\right)$, which were harvested at three different ages $(120,150 \& 180 \mathrm{DAT})$ and two different time sessions of morning and evening. A. paniculata accessions from different ages were able to be differentiated by the intensities of andrographolide (binned at $0.74 \mathrm{ppm}$ ) and deoxyandrographolide (binned at $0.62 \mathrm{ppm})$. The significance $(p<0.05)$ difference between the samples at different age for each accession was conducted by One-Way ANOVA using MINITAB 14. Morning and evening session data for 120, 150 \& 180 DAT of three different accessions ( $\mathrm{P}, \mathrm{T}$ and $\mathrm{H}$ ) were combined in this relative quantification analysis.

The concentration of andrographolide at 180 DAT of $\mathrm{P}$ accession was significantly higher $(p<0.05)$ as compared to 120 and 150 DAT as shown in Figure 3(a). Similarly, $\mathrm{T}$ accession of the harvesting age at $180 \mathrm{DAT}$ showed the highest concentration of andrographolide (Figure 3(b)), but with no significant difference when compared to 120 and $150 \mathrm{DAT}$. The highest concentration of andrographolide for $\mathrm{H}$ accession was observed at 150 DAT instead of 180 DAT with insignificant difference between the three 


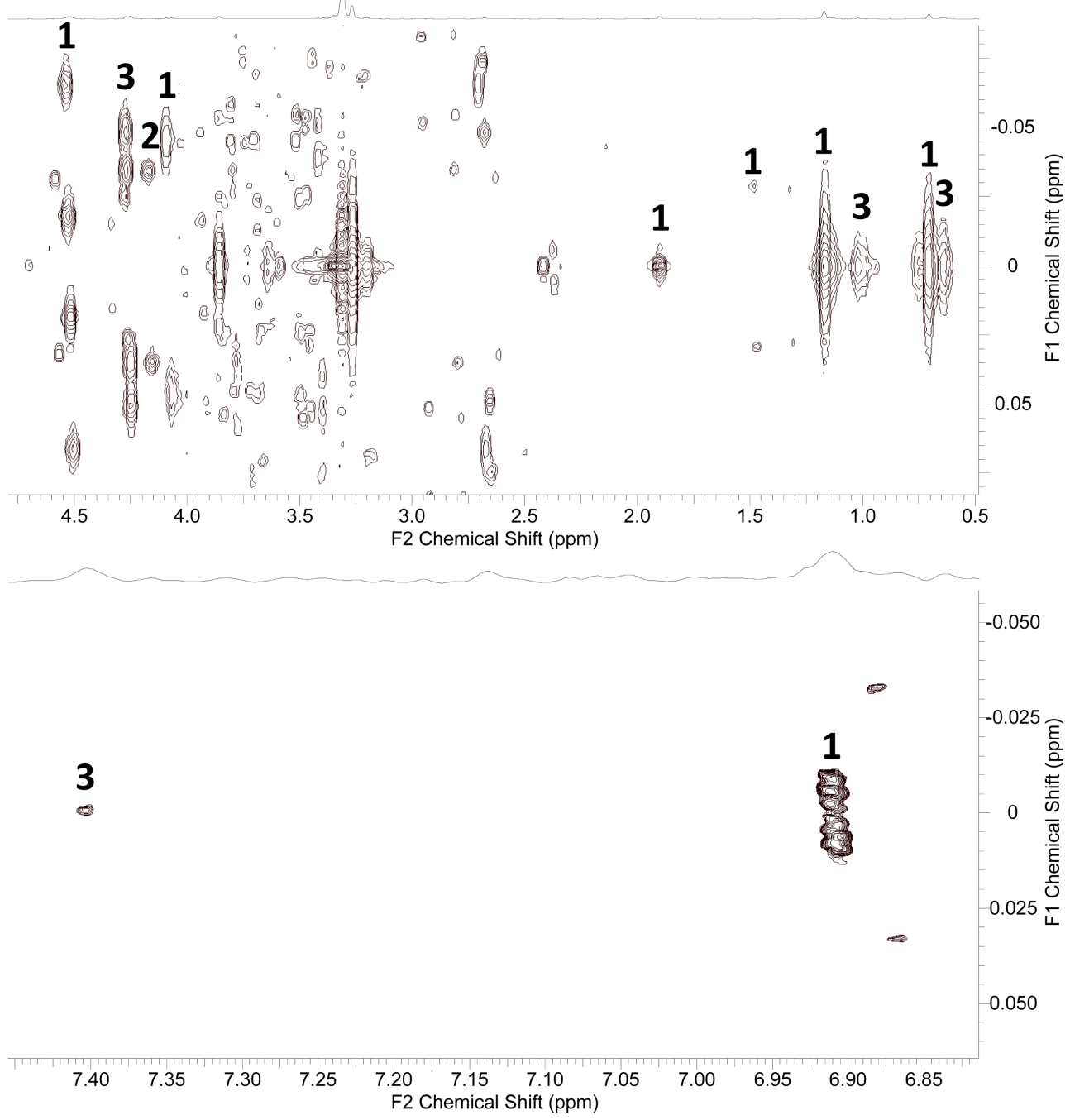

Figure 2. ${ }^{1} \mathrm{H}-{ }^{1} \mathrm{H} J$-resolved spectra of the Andrographis paniculata extract in the region $\delta 0.50$ to 7.45 . The observed signal as follows: 1 -andrographolide; 2-deoxyandrographolide; 3 -neoandrographolide.

DATs (Figure 3(c)) (Table 1). The concentration of neoandrographolide as in Figures 3(a)-(c) was observed to be more abundant in 180 DAT harvesting age for $\mathrm{H}$ and $\mathrm{T}$ accessions, whereas 150 DAT harvesting age for $\mathrm{P}$ accession. However, the $p$ value exhibited no significance between all DATs for each accession (Table 1).

\subsection{Anti-Inflammatory Activity of A. paniculata}

Investigation on the in vitro anti-inflammatory effect of the $A$. paniculata accessions methanol crude extracts was carried out via nitric oxide assay. $A$. paniculata has been used traditionally for inflammation since ages [16]. Thus, based on the claims, this is an attempt made in identifying the possible metabolite changes due to the harvesting time and age variations in relation to the anti-inflammation activity. The correlation of the quantitative $\mathrm{IC}_{50}$ values from NO assay with the data of FT-IR and FT-NMR has paved the way in identifying the possible biomarkers. 
Since the $A$. paniculata leaf extracts have shown promising results at the screening stage, the $\mathrm{IC}_{50}$ values were then further determined as summarized in Table 2 and Figure 4 . The potential of the $A$. paniculata crude extracts as anti-inflammatory agents
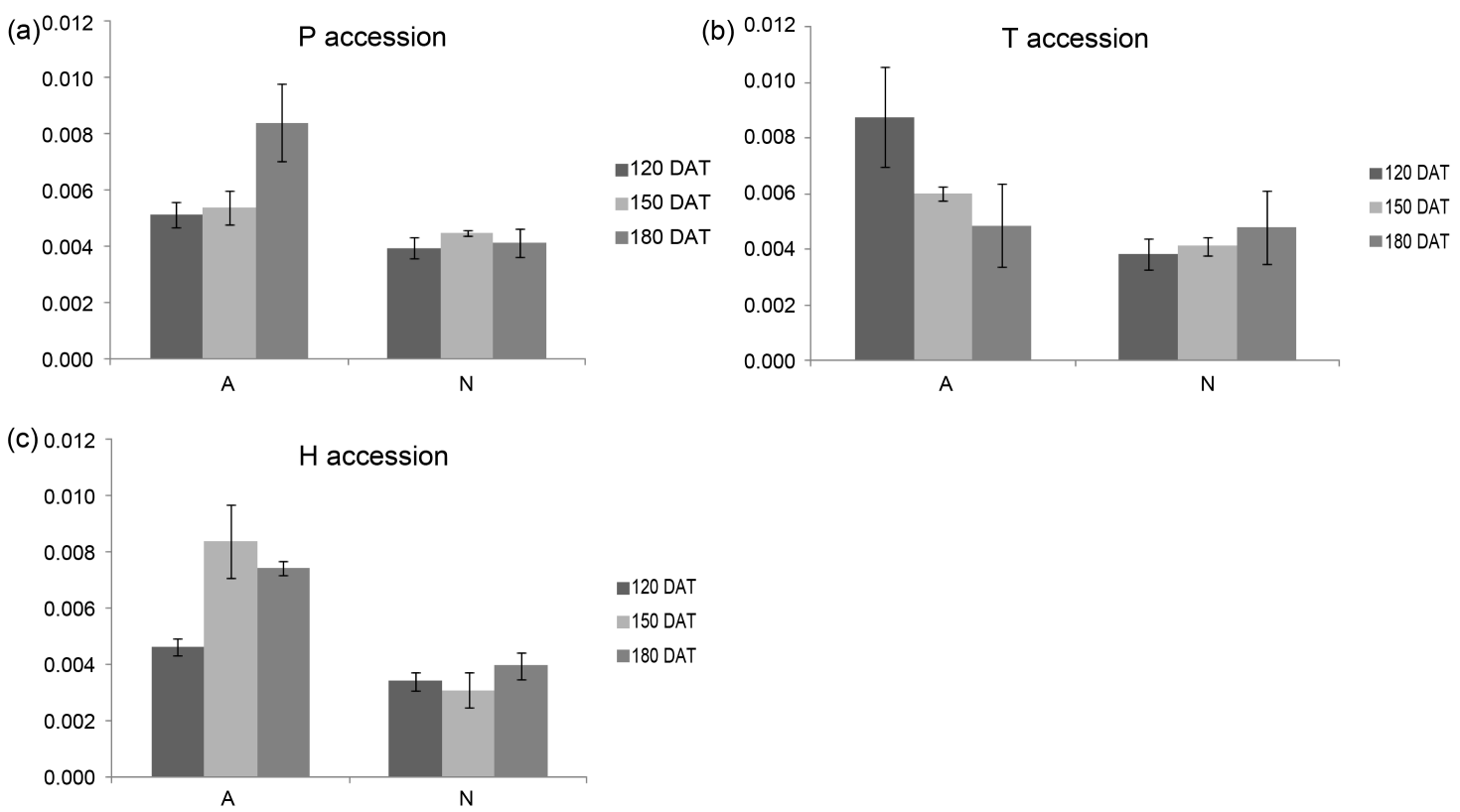

Figure 3. Relative quantification of identified compounds in A. paniculata leaves extract (andrographolide (A) and neoandrographolide $(\mathrm{N})$ ) based on the mean peak area of the ${ }^{1} \mathrm{H}$ NMR signals from three extractions associated with the identified compounds. Chemical shifts $(\delta \mathrm{H})$ used for the relative quantification are andrographolide at 0.74 and neoandrographolide at 0.62 . $Y$-axis expresses the relative peak area of corresponding signal to that of internal standard (TSP): (a) P accession (b) T accession (c) $\mathrm{H}$ accession.
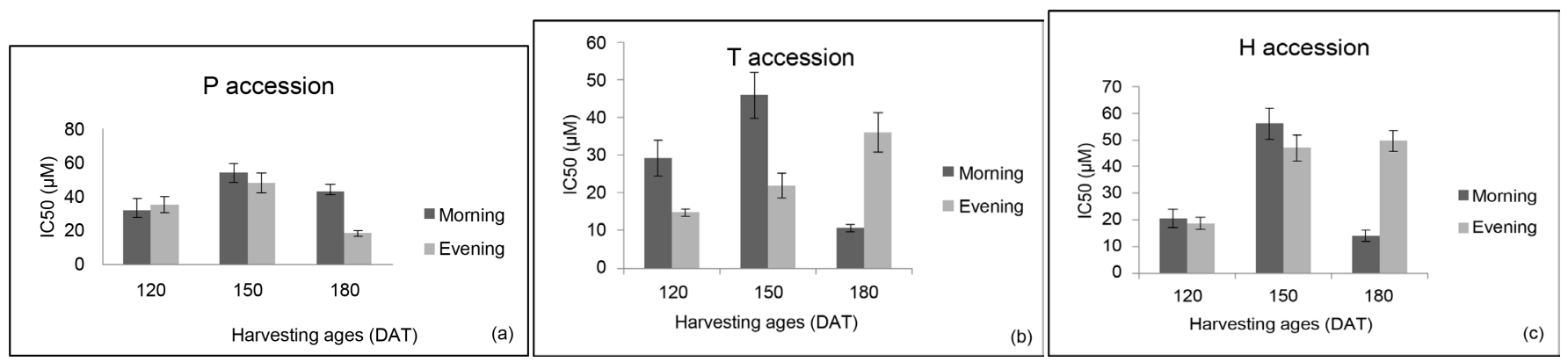

Figure 4. The IC50 for each accession of $A$. paniculata leaf: (a) P accession (b) T accession (c) H accession.

Table 2. The $\mathrm{IC}_{50}$ values of all accessions for NO assay.

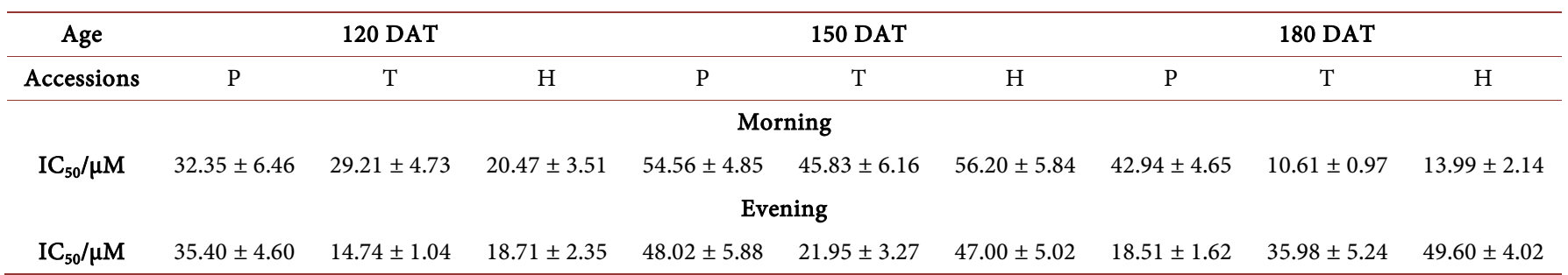

Standard: $\mathrm{IC}_{50}$ curcumin $=14.69 \mu \mathrm{M} ; \mathrm{P}=$ Pasir Puteh, $\mathrm{T}$ = Tanjung Ipoh, $\mathrm{H}=$ Harapan. 

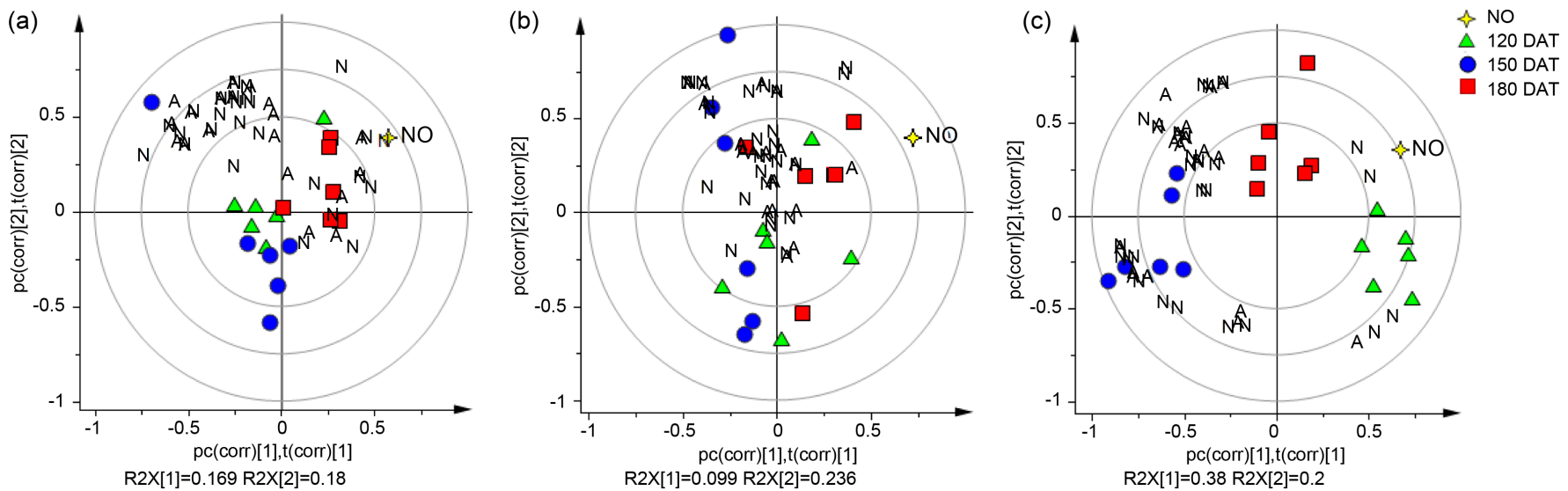

Figure 5. The bi-plot of FTIR data obtained from PLS describing the variation between harvesting ages at 120,150 and 180 DAT of different accession of A paniculata: (a) P accession (b) T accession (c) H accession. (Abbreviations: A, andrographolide; D, deoxyandrographolide; $\mathrm{N}$, neoandrographolide). 
three $A$. paniculata accessions (P, T and $\mathrm{H}$ ) correlated to $\mathrm{NO}$ assay was accomplished (Figure 5). Among the three accessions, P showed that 180 DAT harvesting age was strongly correlated with the NO inhibition activity (Figure 5(a)).

The other two harvesting ages were projected to the negative side of the plot by PC1 which was opposite of the NO. The peak signals were characterized as those belonging to the major compounds of andrographolide and neoandrographolide. The same data distribution was observed over the ellipse for the $\mathrm{T}$ accession of different harvesting ages model (Figure 5(b)). The closest harvesting age to the NO inhibitory property was 180 DAT. This group was projected nearer to the NO in the positive quadrant of both PC1 and PC2.

There was a different observation for $\mathrm{H}$ accession, whereby all the three harvesting ages were well-separated in groups (Figure 5(c)). The 180 DAT was well discriminated from the other two harvesting ages in the positive side of PC2 which gave 180 DAT as the nearest to having NO inhibition property.

\subsection{Correlation between Anti-Inflammatory and Metabolite Variation Using NMR Spectroscopy}

A. paniculata samples were analyzed with NMR spectrometer to support the data obtained from FTIR analysis. The PLS loading bi-plot data gave 180 DAT of $\mathrm{P}$ and T accessions as well separated from 120 and 150 DAT by PC1 (Figure 6(a) and Figure 6(b)), whereby the 180 DAT of P and T were strongly correlated to NO activity. The characteristic of 180 DAT was observed to be having higher level of the major compounds of andrographolide, neoandrographolide and deoxyandrographolide which could be correlated to the NO inhibition property. This is in agreement with the obtained anti-inflammatory activity data in which exhibited that the $180 \mathrm{DAT}$ of $\mathrm{P}$ and $\mathrm{T}$ accessions gave the lowest $\mathrm{IC}_{50}$ value for NO. Other metabolites that contributed to the clustering were carbohydrates and amino acids which are shown in the loading bi-plot of Figure 6(a) and Figure 6(b).
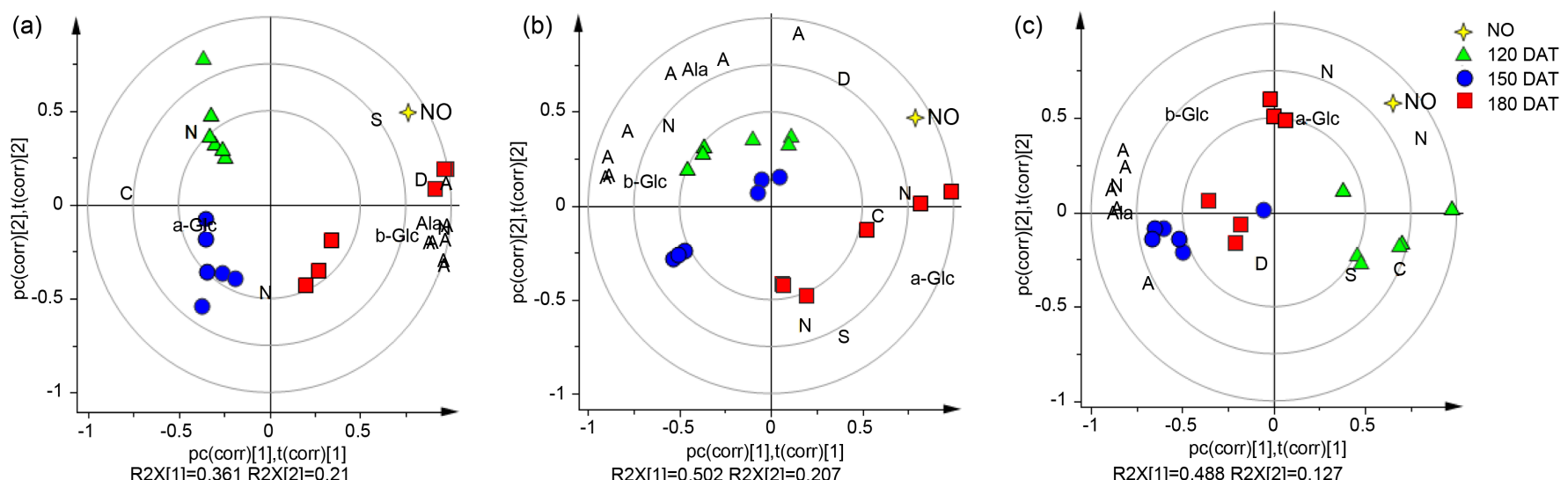

Figure 6. The bi-plot of NMR data obtained from PLS describing the variation between harvesting ages at 120,150 and 180 DAT of different accession of $A$. paniculata: (a) P accession (b) T accession (c) H accession (Abbreviations. A, andrographolide; D, deoxyandrographolide; $\mathrm{N}$, neoandrographolide; a-Glc, $\alpha$-glucose; $\mathrm{B}-\mathrm{Glc}, \beta$-glucose; $\mathrm{S}$, sucrose; Ala, alanine; $\mathrm{C}$, choline; NO, nitric oxide). 
$\mathrm{H}$ accession gave a different observation wherein both 120 and 180 DAT were close to NO assay compared to $150 \mathrm{DAT}$ as shown in Figure 6(c). Even though both of the DATs of 120 and 180 situated close to the NO activity, the clustering of these two could be established as 120 DAT was closest to NO by PC1 whereas 180 DAT by PC2 with 180 DAT having the highest NO inhibition. Metabolites that contributed to this separation were andrographolide, neoandrographolide and carbohydrates with higher content of neoandrographolide and amino acids in 120 DAT.

The correlation between anti-inflammatory and metabolite variations among the three different harvesting ages of $\mathrm{P}, \mathrm{T}$ and $\mathrm{H}$ accession using NMR spectroscopy in this study is agreeing with the data obtained from the FTIR analysis. Both of the spectroscopic data are in congruent when correlated to the NO inhibition activity.

\subsection{Permutation Test}

PLS model validation was performed by checking the $Q^{2}$ and $R^{2}$ accumulative means after cross validation and permutation test with 100 permutations (Figure 7). According to Eriksson et al. (2006), the permutation test will generate $Q^{2}$ value higher than 0.5, $R^{2}$ value must always be larger than the value of $Q^{2}$ for a good model [19]. Thus, with
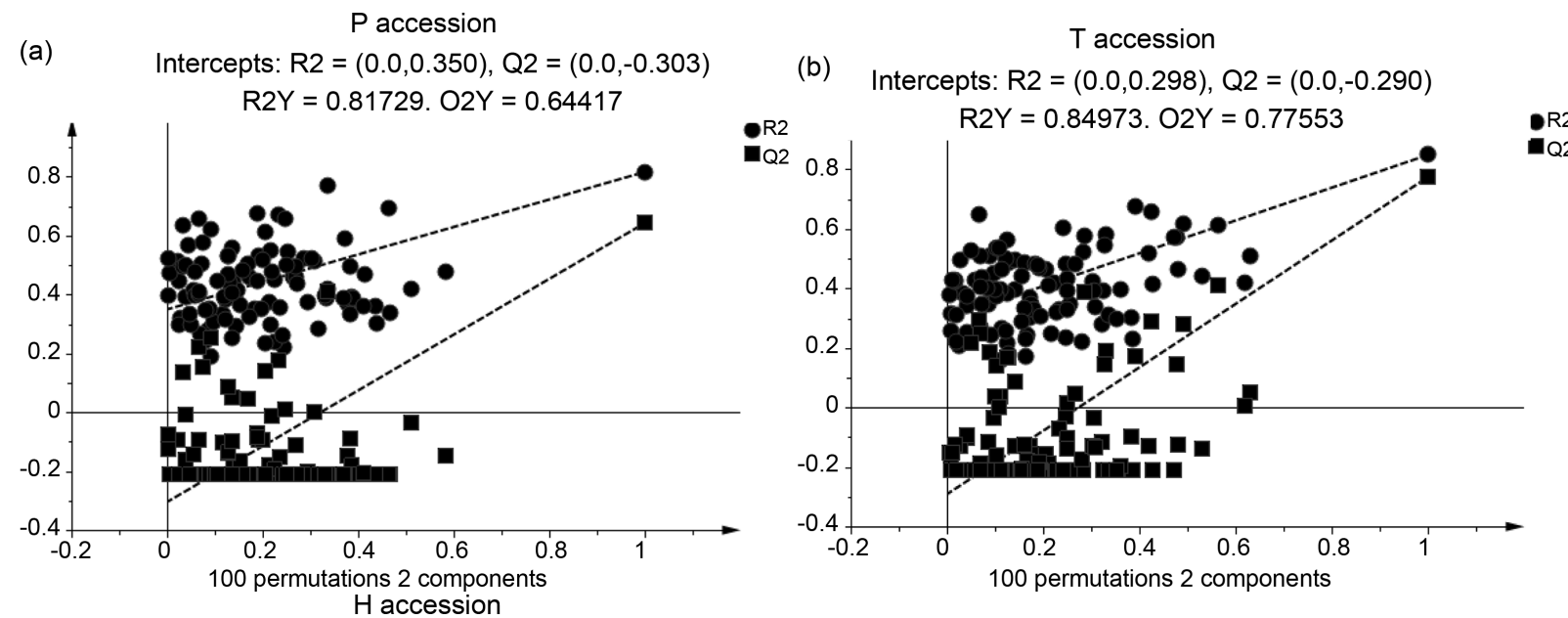

(c) Intercepts: R2 $=(0.0,0.447), Q 2=(0.0,-0.189)$

$\mathrm{R} 2 \mathrm{Y}=0.75833 . \mathrm{O} 2 \mathrm{Y}=0.57511$

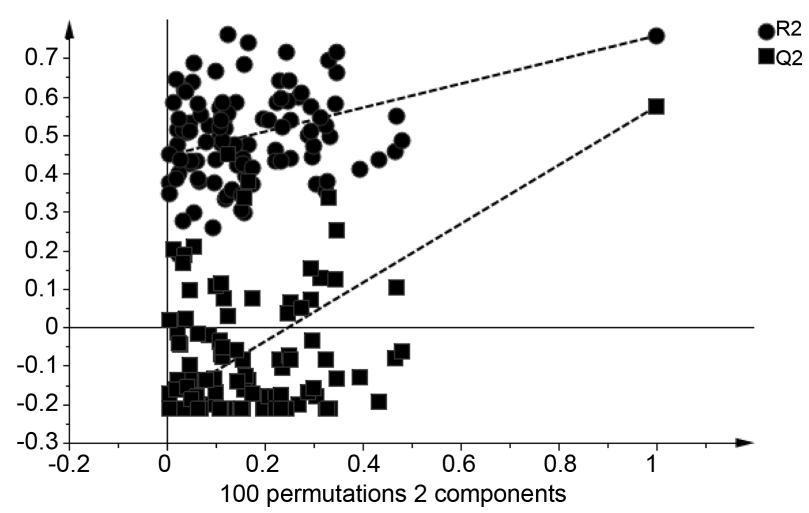

Figure 7. The PLS validation for each harvesting age model for: (a) P accession; (b) T accession and (c) $\mathrm{H}$ accession. 
the higher significance and lower variation of the NMR-adopted-permutation has helped each model to be validated as a good model. In the current study, the $Q^{2}$ and $R^{2}$ values were higher than 0.5 (P accession: $R^{2} Y=0.75833, Q^{2} Y=0.71733$; $\mathrm{T}$ accession: $R^{2} Y=$ 0.84973, $Q^{2} Y=0.77553$; H accession: $\left.R^{2} Y=0.75833, Q^{2} Y=0.57511\right)$ which suggested that all of the models meet the criteria of the validation and prediction performances.

\section{Conclusion}

Utilizing the FTIR and NMR-metabolomics approach has provided a rapid way to study the metabolites variation of $A$. paniculata in relationship to its different harvesting ages $\&$ time with a biological activity of anti-inflammation. The PLS allowed the separation and correlation between the NO assay with the phytochemical present due to the effects of different harvesting ages and times. $\mathrm{P}(11,341), \mathrm{T}(11,248)$ and $\mathrm{H}(11,265)$ accessions exhibited $180 \mathrm{DAT}$ as the best harvesting age possessing the highest anti-inflammatory activity. The accessions at this age also contained higher level of the major compounds which could be attributed to the anti-inflammatory capacity.

\section{Acknowledgements}

Our appreciation goes to Professor Dr. Johnson Stanslas, Pharmacotherapeutics Unit, Department of Medicine, Faculty of Medicine and Health Sciences, Universiti Putra Malaysia for providing the andrographolide, deoxyandrographolide and neoandrographolide. The authors also thank Mr. Tajuddin Abd Manap (Biodiversity Unit, Institute of Bioscience, UPM) and Mr. Mohd Norhaizan Saliudin (Department of Crop Science, Faculty of Agriculture, UPM) for guiding in the plant cultivation. This work was supported by Research University Grant Schemes (RUGS) of Universiti Putra Malaysia (grant number 91,968) and Exploratory Research Grant Scheme (ERGS) of Ministry of Higher Education Malaysia (grant number 5,527,124).

\section{References}

[1] Vijaykumar, K., Murthy, P.B., Kannababu, S., Syamasundar, B. and Subbaraju, G.V. (2007) Estimation of Andrographis paniculata Herb, Extracts and Dosage Forms. International Journal of Applied Science and Engineering, 5, 27-39.

[2] Gupta, P.P., Tandon, J.S. and Patnaik, G.K. (1998) Antiallergic Activity of Andrographolides Isolated from Andrographis paniculata (Burm. F.) Wall. Pharmaceutical Biology, 36, 72-74. https://doi.org/10.1076/phbi.36.1.72.4620

[3] Habtemariam, S. (1998) Andrographolide Inhibits the Tumor Necrosis Factor- $\alpha$ Induced Upregulation of ICAM-1 Expression and Endothelial-Monocyte Adhesion. Phytotherapy Research, 12, 37-40. https://doi.org/10.1002/(SICI)1099-1573(19980201)12:1<37::AID-PTR186>3.0.CO;2-N

[4] Handa, S.S. and Sharma, A. (1990) Hepatoprotective Activity of Andrographolide from Andrographis paniculata against Carbon Tetrachloride. Indian Journal of Medical Research, 92, 276-283.

[5] Matsuda, T., Kuroyanagi, M., Sugiyama, S., Umehara, K., Ueno, A. and Nishi, K. (1994) Cell Differentiation-Inducing Diterpenes from Andrographis paniculata Nees. Chemical and 
Pharmaceutical Bulletin, 42, 1216-1225. https://doi.org/10.1248/cpb.42.1216

[6] Puri, A., Saxena, R., Saxena, R.P. and Saxena, K.C. (1993) Immunostimulant Agents from Andrographis paniculata. Journal of Natural Products, 56, 995-999.

https://doi.org/10.1021/np50097a002

[7] Trivedi, N.P. and Rawal, U.M. (1998) Effect of Aqueous Extract of Andrographis paniculata on Liver Tumor. Indian Journal of Pharmacology, 30, 318-322.

[8] Zhang, X.F. and Tan, B.K. (2000) Antihyperglycaemic and Anti-Oxidant Properties of Andrographis paniculata in Normal and Diabetic Rats. Clinical and Experimental Pharmacology and Physiology, 27, 358-363. https://doi.org/10.1046/j.1440-1681.2000.03253.x

[9] Parichatikanond, W., Suthisisang, C., Dhepakson, P. and Herunsalee, A. (2010) Study of Anti Inflammatory Activities of the Pure Compounds from Andrographis paniculata (burm.f.) Nees and Their Effects on Gene Expression. International Immunopharmacology, 10, 13611373. https://doi.org/10.1016/j.intimp.2010.08.002

[10] Chandrasekaran, C.V., Thiyagarajan, P., Deepak, H.B. and Agarwal, A. (2011) In Vitro Modulation of LPS/Calcimycin Induced Inflammatory and Allergic Mediators by Pure Compounds of Andrographis paniculata (King of Bitters) Extract. International Immunopharmacology, 11, 79-84. https://doi.org/10.1016/j.intimp.2010.10.009

[11] Kumar, A., Amit, A., Sujatha, M., Murali, B. and Anand, M.S. (2002) Effect of Aging on Andrographolide Content in Kalmegh. Journal of Natural Remedies, 292, 179-181.

[12] Yusof, N.A., Isha, A., Ismail, I.S., Khatib, A., Shaari, K., Abas, F. and Rukayadi, Y. (2015) Infrared-Metabolomics Approach in Detecting Changes in Andrographis paniculata Metabolites Due to Different Harvesting Ages and Times. Journal of the Science of Food and Agriculture, 95, 2533-2543.

[13] Kim, H.Y., Choi, Y.H. and Verpoorte, R. (2010) NMR-Based Metabolomic Analysis Plants. Nature Protocols, 5, 536-549. https://doi.org/10.1038/nprot.2009.237

[14] Lee, K., Ab. Aziz, F., Syahida, A., Abas, F., Shaari, K., Israf, D. and Lajis, N. (2009) Synthesis and Biological Evaluation of Curcumin-Like Diarylpentanoid Analogues for Anti-Inflammatory, Antioxidant and Anti-Tyrosinase Activities. European Journal of Medicinal Chemistry, 44, 3195-3200. https://doi.org/10.1016/j.ejmech.2009.03.020

[15] de las Heras, B., Abad, M., Silván, A., Pascual, R., Bermejo, P., Rodriguez, B. and Villar, A. (2001) Effects of Six Diterpenes on Macrophage Eicosanoid Biosynthesis. Life Sciences, 70, 269-278. https://doi.org/10.1016/S0024-3205(01)01402-3

[16] Joselin, J. and Jeeva, S. (2014) Andrographis paniculata: A Review of Its Traditional Uses, Phytochemistry and Pharmacology. Medicinal \& Aromatic Plants, 3, 1-15.

[17] Maisuthisakul, P., Pasuk, S. and Ritthiruangdej, P. (2008) Relationship between Antioxidant Properties and Chemical Composition of Some Thai Plants. Journal of Food Composition and Analysis, 21, 229-240. https://doi.org/10.1016/j.jfca.2007.11.005

[18] Kim, J., Choi, J.N., Ku, K.M., Kang, D., Kim, J.S., Park, J.H.Y. and Lee, C.H. (2011) A Correlation between Antioxidant Activity and Metabolite Release during the Blanching of Chrysanthemum coronarium L. Bioscience, Biotechnology, and Biochemistry, 75, 674-680. https://doi.org/10.1271/bbb.100799

[19] Eriksson, L. (2006) Multi- and Megavariate Data Analysis. Umetrics AB, Umeå, Sweden. 
Submit or recommend next manuscript to SCIRP and we will provide best service for you:

Accepting pre-submission inquiries through Email, Facebook, LinkedIn, Twitter, etc. A wide selection of journals (inclusive of 9 subjects, more than 200 journals)

Providing 24-hour high-quality service

User-friendly online submission system

Fair and swift peer-review system

Efficient typesetting and proofreading procedure

Display of the result of downloads and visits, as well as the number of cited articles

Maximum dissemination of your research work

Submit your manuscript at: http://papersubmission.scirp.org/

Or contact jbm@scirp.org 